\title{
Implications of precordial ST segment depression during acute inferior myocardial infarction Arteriographic and ventriculographic correlations during the acute phase
}

\author{
MARC COHEN, HEINER BLANKE, KARL R KARSH, JAMES HOLT, PETER RENTROP \\ From the Division of Cardiology, Department of Medicine, Mount Sinai School of Medicine, and the Mount Sinai \\ Hospital, New York, USA
}

SUMMARY Thirty two patients presenting with acute transmural inferior wall myocardial infarction underwent cardiac catheterisation and angiography within 12 hours of the onset of symptoms. Twelve lead electrocardiograms performed within one hour of catheterisation showed ST segment depression in the anterior precordial leads in addition to inferior wall changes in 17 patients and no ST segment changes in the anterior leads in 15. When the clinical, arteriographic, and ventriculographic variables were compared between the two groups no significant differences were noted with regard to age, sex, risk factors for coronary disease, duration of symptoms before angiography, Killip class, number of inferior leads with ST segment elevation, or initial serum creatine kinase activity. The extent of coronary artery disease as well as the prevalence of severe disease in the left anterior descending artery were similar for both groups. Biplane left ventriculography showed no significant differences between the two groups with regard to global ejection fraction or to the prevalence of posterolateral or anterior segmental wall motion abnormalities.

Patients presenting with acute transmural inferior infarction often have ST segment depression in the anterior precordial leads. Investigators have suggested that, in contrast to patients with inferior infarction without additional precordial ST depression, patients with anterior changes have larger infarctions, more extensive coronary artery disease, and a poorer long term prognosis. ${ }^{1-5}$ Other investigators, however, found no significant differences between these two groups of patients. ${ }^{6-10}$ These conflicting results are probably due to differences in patient selection and the timing and method of evaluating the electrocardiographic and angiographic changes. In order to minimise the biases introduced by $(a)$ evaluating coronary anatomy and ventricular function days or months after the acute phase of infarction and (b) non-randomised patient selection we studied patients

Requests for reprints to Dr Marc Cohen, Division of Cardiology, Department of Medicine, Mount Sinai Hospital, 1 Gustave Levy Place, New York, New York 10029, USA.

Accepted for publication 28 June 1984 with inferior wall myocardial infarction extracted from a larger pool of consecutive patients who underwent cardiac catheterisation with 12 hours of the onset of symptoms of infarction. In addition, the electrocardiographic and angiographic data were obtained within one hour of each other. Lastly, biplane ventriculography, the most sensitive method currently available for evaluating segmental wall motion abnormalities and ejection fraction, was used in all patients. We compared the differences between patients with and without precordial ST segment depression during acute inferior wall infarction with regard to their clinical, arteriographic, and ventriculographic characteristics.

\section{Patients and methods}

\section{STUDY POPULATION}

Patients presenting with complaints of acute chest pain suggestive of myocardial ischaemia were screened for possible immediate cardiac catheterisation and coronary arteriography. Inpatients requiring 
emergency cardiac consultation for acute chest pain were screened in a similar fashion. No patients with (a) valvar or congenital heart disease, (b) active psychiatric disorder or alcohol addiction, or (c) contraindication to thrombolytic treatment underwent immediate catheterisation. No patients were excluded from immediate catheterisation because of hypotension or cardiogenic shock. Patients with persistent ischaemic chest pain lasting longer than $\mathbf{3 0}$ minutes suggestive of acute myocardial infarction underwent immediate cardiac catheterisation after informed consent was obtained. In 52 patients who underwent immediate catheterisation the diagnosis of acute myocardial infarction was established retrospectively based on increased creatine phosphokinase and creatine phosphokinase MB activity to at least twice the upper limit of normal. Thirty five of these patients had electrocardiographic evidence of transmural inferior wall infarction. Three of these 35 patients, however, had previous anterior wall infarction and were excluded from this study. The remaining 32 patients constitute the study population. All patients received the same medical regimen-that is, prophylactic intravenous lignocaine and morphine sulphate for analgesia - until after baseline electrocardiography and angiography had been performed.

\section{ELECTROCARDIOGRAM}

All patients had a standard 12 lead electrocardiogram recorded on admission and within one hour of cardiac catheterisation. All electrocardiograms were recorded on either three or six channel recorders using a paper speed of $50 \mathrm{~mm} / \mathrm{s}$ and including $1 \mathrm{mV}$ standardisation. $Q$ waves of longer than $0.04 \mathrm{~s}$ or $\mathbf{0 . 0 3} \mathrm{s}$ in association with a $Q: R$ ratio $1: 3$ were considered to be abnormal. In lead $V 1, R>S$ and $R>5 \mathrm{~mm}$ was considered to be pathological. ST segment elevation of $\geqslant 1 \mathrm{~mm}$ above the baseline (as defined by the preceding TP segment) occurring $0.02 \mathrm{~s}$ after the end of the QRS complex in any lead was considered to be abnormal. The sum of the number of inferior leads in which abnormal ST segment elevation was present was calculated for each patient. ST segment depression $\geqslant 1 \mathrm{~mm}$ occurring $0.88 \mathrm{~s}$ after the $\mathrm{J}$ point in any lead was considered to be significant. The electrocardiogram recorded within one hour before cardiac catheterisation was used for the analysis of all the electrocardiographic variables in this study.

In 32 patients acute inferior myocardial infarction was diagnosed on the basis of abnormal $\mathrm{Q}$ waves or ST segment elevation in at least two of the following leads: II, III, or aVF. In five patients true posterior wall infarction was diagnosed on the basis of $R>S$ and $R>5 \mathrm{~mm}$ in lead V1 in the presence of an upright T wave. ${ }^{11}$
No patient in the study group was taking digoxin or had left ventricular hypertrophy, intraventricular conduction abnormality, right bundle branch block, or left bundle branch block. Seventeen of the 32 patients (group 1) had precordial ST segment depression in at least two of the following leads: V1-V4 and 15 did not (group 2). Four patients in group 1 had electrocardiographic evidence of a true posterior myocardial infarction in contrast to only one patient in group 2.

\section{ANGIOGRAPHY AND VENTRICULOGRAPHY}

The technique of angiography during the acute phase of myocardial infarction has been described previously. ${ }^{12}$ The degree of luminal narrowing in the coronary arteries was assessed by the method of Gensini. ${ }^{13}$ Stenosis in the coronary artery was considered to be significant if the luminal diameter was reduced by $>70 \%$. Wall motion abnormalities were described according to the method of Herman et al. ${ }^{14}$ The specific myocardial segments were identified from the right anterior oblique and left anterior oblique ven triculograms in a manner similar to that in the coronary artery surgery study. ${ }^{15}$

\section{Determination of infarct vessel}

The infarct vessel was determined by the following angiographic criteria: coronary artery disease resulting in a total or subtotal occlusion of one vessel (29 patients) and $>90 \%$ stenosis in two or three vessels (three patients). In these three patients, there were arteriographic features suggesting acute thrombus in one of the vessels. The arteriographic findings of persistent staining of intraluminal material with dye, or intraluminal filling defects completely surrounded by dye, were considered to suggest a thrombus. ${ }^{16} 17$ Wall motion abnormalities were present in the areas subtended by the infarct related vessel in all 32 patients.

\section{STATISTICS}

Continuous variables are expressed as the mean (standard deviation). Differences between the means of independent continuous variables were evaluated by a $t$ test. Differences between discrete variables were evaluated by a $\chi^{2}$ test. Fisher's exact test was used for small samples in fourfold tables. A probability value of $<0.05$ was considered to be significant. Statistical comparisons were performed between groups 1 and 2 before and after those patients with electrocardiographic evidence of true posterior infarction had been excluded from both groups. Inferences, based on the data presented, are made with some reservation because of the relatively small sample size. 
Table Clinical and angiographic characteristics

\begin{tabular}{lll}
\hline & Group $I(n=17)$ & Group $2(n=15)$ \\
\hline Mean (SD) age (yr) & $56 \cdot 7(8 \cdot 9)$ & $53 \cdot 4(11 \cdot 1)$ \\
Sex (M/F) & $16 / 1$ & $13 / 2$ \\
\% of patients with diabetes or hypertension & $8 \%$ & $6 \%$ \\
Duration from onset of symptoms to angiography & & \\
(h, mean (SD)) & $4 \cdot 7(4 \cdot 1)$ & $6 \cdot 5(6 \cdot 0)$ \\
No of patients in Killip class: I or II & 15 & 15 \\
Number-of inferior leads with ST or IV & 2 & 0 \\
Initial creatine kinase activity (IU/l, mean (SD)) & $2 \cdot 7(0 \cdot 8)$ & $157(264)$ \\
Infarct related vessel (No of patients): & $168(155)$ & 13 \\
$\quad$ Right coronary & 13 & 2 \\
Circumflex & 4 & 6 \\
Extent of coronary artery disease: & 7 & 7 \\
$\quad$ One vessel & 6 & 2 \\
Two vessel & 4 & $56 \cdot 1(12 \cdot 0)$ \\
Three vessel & $53 \cdot 4(9 \cdot 8)$ & \\
Left ventricular ejection fraction (\%), mean (SD) & &
\end{tabular}

\section{Results}

CLINICAL AND ANGIOGRAPHIC CHARACTERISTICS There were no significant differences detected between the two groups of patients with respect to age, sex, or the prevalence of diabetes mellitus or hypertension (Table). The duration from the onset of symptoms of acute infarction to cardiac angiography was $4 \cdot 7(4 \cdot 1)$ h for group 1 and $6 \cdot 5(6.0)$ h for group $2(\mathrm{NS})$. Clinical evaluation of the patients just before angiography showed no significant differences with respect to Killip class or initial serum creatine kinase activity. Although patients in group 1 had a higher number of leads with ST segment elevation than those in group

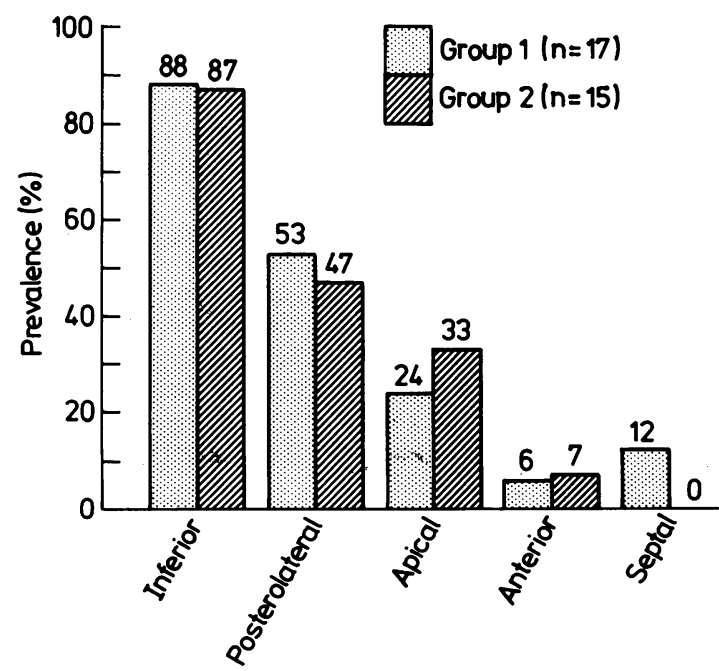

Figure Prevalence of wall motion abnormalities in myocardial segments in patients with precordial ST segment depression (group 1) compared with those without (group 2).
2 , this difference did not reach statistical significance.

There were no significant differences between the two groups with respect to the extent of coronary artery disease or to the left ventricular ejection fraction. Two patients in both groups had a stenosis $\geqslant 90 \%$ in their left anterior descending artery. All patients in both groups, except for one in group 1, had a right dominant coronary circulation.

\section{WALL MOTION ABNORMALITIES}

The segmental wall motion abnormalities seen during biplane ventriculography are compared for both groups of patients in the Figure. Most patients, in both groups, had inferior and posterolateral wall motion abnormalities. Nevertheless, no significant differences were observed with regard to the frequency of posterolateral involvement. In addition, both groups of patients had comparably low frequencies of anterior and or septal wall motion abnormalities.

\section{Discussion}

Several reports have suggested that patients with precordial ST segment depression in acute inferior myocardial infarction were at higher risk than those with inferior wall infarction but without precordial ST segment depression. ${ }^{1-5}$ The investigations of Salcedo et al and of Jennings et al suggested that patients with precordial ST segment depression were more likely to have additional coronary disease in the left anterior descending artery and anterior wall ischaemia. ${ }^{2} 18$ These studies, however, were based on patients who underwent cardiac catheterisation several weeks or months after their inferior infarction for progressive or recurrent symptoms. Since patients with postinfarction symptoms are more likely to have multivessel disease this selection bias may have affected their results. 
Our study was based on arteriographic and ventriculographic data obtained during the acute phase of inferior infarction in consecutive patients. In this random population, in contrast to the studies mentioned above, we did not observe any significant differences in the prevalence of left anterior descending disease. In addition, both groups of patients had a comparably low prevalence of anterior or septal wall motion abnormalities.

Other studies have suggested that precordial ST segment depression in inferior infarction is a marker for a more extensive infarction. ${ }^{3419}$ Gibson et al, using radionuclide techniques in the late hospital phase after myocardial infarction, noted a lower left ventricular ejection fraction in patients with precordial ST segment depression. ${ }^{4}$ They attributed this finding to larger inferior and inferoapical infarctions.

In our study calculation of left ventricular ejection fraction based on biplane ventriculography obtained within one hour of the electrocardiogram showed no significant differences between our two groups. Our findings agree with the studies of Croft et al and O'Neill et al, who also found no significant difference in ejection fraction between patients with and without precordial ST segment depression. ${ }^{6} 20$

Myers et al correlated precordial ST segment depression in acute inferior infarction with pathological evidence of posterolateral extension of inferior wall infarction. ${ }^{21}$ More recently, Goldberg et al studied 25 patients with acute inferior infarction using radionuclide techniques during the acute phase of infarction. ${ }^{22}$ Their observations suggested that patients with precordial ST segment depression had a much higher prevalence of posterolateral wall motion abnormalities than those with inferior infarction without precordial ST segment depression. Eleven of the 14 patients in their group with precordial ST segment depression, however, met classical electrocardiographic criteria for additional posterior infarction in contrast to only four of 11 patients in their group without precordial ST segment depression. The uneven distribution of patients with additional electrocardiographic evidence of posterior infarction between their two groups of patients may explain the greater prevalence of posterolateral wall motion abnormalities in their patients with pr-cordial ST segment depression.

Our study, in which patients with electrocardiographic evidence of posterior infarction were more evenly distributed between the two study groups, did not show a significantly higher prevalence of posterior wall motion abnormalities in the patients with precordial ST segment depression. In this regard our observations agree with the earlier work of Croft et al. 6

Since several of our patients underwent interventions during the acute phase of their infarction this study cannot definitely evaluate differences in out- come or prognosis between the two groups of patients. There is, however, a close relation between ejection fraction in the acute phase of myocardial infarction and long term prognosis. ${ }^{23}$ The data on ejection fraction in the early phase may therefore have some prognostic significance.

Based on the correlations that were obtained during the early hours of infarction this study suggests that patients with anterior ST segment depression do not constitute a subset at increased risk. Clinical trials with larger patient populations, using angiography during the acute phase of infarction, should provide an even more definitive evaluation.

\section{References}

1 Miller RR, DeMaria AN, Vismara LE, et al. Chronic stable inferior myocardial infarction: unsuspected harbinger of high-risk proximal left coronary arterial obstruction amenable to surgical revascularization. Am $\mathcal{J}$ Cardiol 1977; 39: 954-60.

2 Salcedo JR, Baird MG, Chambers RJ, Beanlands DS. Significance of reciprocal S-T segment depression in anterior precordial leads in acute inferior myocardial infarction: concomitant left anterior descending coronary artery disease? Am $\mathcal{f}$ Cardiol 1981; 48: 1003-8.

3 Shah PK, Pichler M, Berman DS, et al. Noninvasive identification of a high risk subset of patients with acute inferior myocardial infarction. Am $\mathcal{F}$ Cardiol 1980; 46: 915-21.

4 Gibson RS, Crampton RS, Watson DD, et al. Precordial ST-segment depression during acute inferior myocardial infarction: clinical, scintigraphic and angiographic correlations. Circulation 1982; 66: 732-41.

5 Haraphongse M, Jugdutt BI, Rossall RE. Significance of precordial ST-segment depression in acute transmural inferior infarction: coronary angiographic findings. Cathet Cardiovasc Diagn 1983; 9: 143-51.

6 Croft CH, Woodward W, Nicod P, et al. Clinical implications of anterior S-T segment depression in patients with acute inferior myocardial infarction. Am $\mathcal{F}$ Cardiol 1982; 50: 428-36.

7 Ferguson DW, Pandian N, Kioschos JM, Marcus ML, White CW. Angiographic evidence that reciprocal STsegment depression during acute myocardial infarction does not indicate remote ischemia: analysis of 23 patients. Am $\mathcal{Y}$ Cardiol 1984; 53: 55-62.

8 Stafford A, Lloyd BL, Thompson PL. Anterior ST segment depression in inferior myocardial infarction: lack $N$ of effect on long-term survival [Abstract]. Circulation 1983; 68 (suppl III): 407.

9 Wasserman AG, Ross AM, Bogaty D, Richardson DW, Hutchinson RG, Rios JC. Anterior ST segment depression during acute inferior myocardial infarction: evi- 0 dence for the reciprocal change theory. Am Heart $f$ 1983; 106: 516-20.

10 Camera EJ, Chandra N, Ouyang P, Gottlieb SH, Shapiro EP. Reciprocal ST change in acute myocardial infarction: assessment by electrocardiography and echocardiography. $f$ Am Coll Cardiol 1983; 2: 251-7.

\section{(a)} 品 (n) 。 言

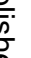
(1) , 
11 Perloff JK. The recognition of strictly posterior myocardial infarction by conventional scalar electrocardiography. Circulation 1954; 30: 706-18.

12 Rentrop KP, Blanke H, Karsch KR, Kaiser H, Köstering $H$, Leitz $K$. Selective intracoronary thrombolysis in acute myocardial infarction and unstable angina pectoris. Circulation 1981; 63: 307-17.

13 Gensini GG. Coronary angiography. Mount Kisko, New York: Futura, 1975; 260.

14 Herman MV, Heinle RA, Klein MD, Gorlin R. Localized disorders in myocardial contraction. $N$ Engl $\mathcal{F}$ Med 1967; 277: 222-32.

15 National Heart, Lung and Blood Institute. Coronary artery surgery study (CASS). Circulation 1981; 63 (suppl I): $1-15$.

16 DeWood MA, Spores J, Notske R, et al. Prevalence of total occlusion during the early hours of transmural myocardial infarction. $N$ Engl $\mathcal{F}$ Med 1980; 303: 897902.

17 Mathey DG, Kuck KH, Tilsner V, Krebber HJ, Bleifeld W. Nonsurgical coronary artery recanalization in acute transmural myocardial infarction. Circulation 1981; 63: 489-97.

18 Jennings K, Reid DS, Julian DG. "Reciprocal" depres- sion of the ST segment in acute myocardial infarction. $\mathrm{Br}$ Med F 1983; 287: 634-7.

19 DeWood MA, Heit J, Spores J, et al. Significance of precordial ST segment depression in acute inferior transmural myocardial infarction: assessment by coronary arteriography and ventriculography during the early hours [Abstract]. Circulation 1982; 66 (suppl II): 182.

20 O'Neill W, Walton J, Colfer HT, et al. Anterior ST segment depression in inferior myocardial infarction: angiographic, ventriculographic, and scintigraphic findings [Abstract]. Circulation 1982; 66 (suppl II): 182.

21 Myers GB, Klein HA, Hiratzka T. Correlation of electrocardiographic and pathologic findings in posterolateral infarction. Am Heart $\mathcal{F} 1949$; 38: 837-62.

22 Goldberg HL, Borer JS, Jacobstein JG, Kluger J, Scheidt SS, Alonso DR. Anterior S-T segment depression in acute inferior myocardial infarction: indicator of posterolateral infarction. Am f Cardiol 1981; 48: 100915.

23 Shah PK, Pichler M, Berman DS, Singh BN, Swan HJC. Left ventricular ejection fraction determined by radionuclide ventriculography in early stages of first transmural myocardial infarction. Am $\mathcal{F}$ Cardiol 1980; 45: 542-6. 